\title{
STUDY OF ONYCHOMYCOSIS AT A TERTIARY CARE HOSPITAL IN SOUTH INDIA
}

Niranjan H. P., N. Padmaja, Priyanka. B. V.

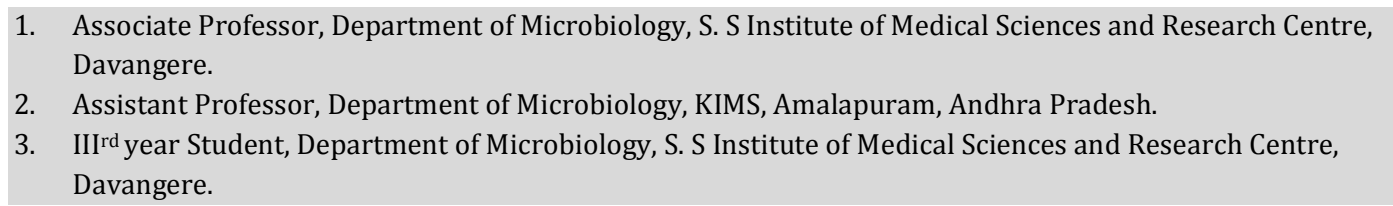

\section{CORRESPONDING AUTHOR}

Dr. Niranjan H.P.,

B-7, Staff Quarters, SSIMS \& RC,

Davangere - 577005.

E-mail: niranjanhp@gmail.com

Ph: +919844821527.

\section{ABSTRACT:}

BACKGROUND: Onychomycosis is common infection in adults and account for $20 \%$ of all nail diseases. The clinical presentation is often confused with other conditions, making laboratory diagnosis and confirmation necessary. Accurate identification of etiological agent is important, as the treatment is different for dermatophytes and non dermatophytes. AIMS AND OBJECTIVES: 1 . To isolate and identify the etiological agents of onychomycosis. 2 . To study the occupational status of the study group. 3. Correlation between nail involvement and sex. METHODOLOGY: To identify the aetiological agents of onychomycosis, the present study was carried out in the department of Microbiology, S. S. Institute of medical sciences and research centre. Nail clippings were collected from a total of 120 clinically suspected patients of onychomycosis attending the outpatient department of Dermatology and Venereology and were processed and identified by standard laboratory techniques. RESULTS: Fungi was demonstrated in 66 (55\%) either by KOH preparation and/or culture. Sixty (50\%) were positive by direct microscopy and 54 (45\%) were culture positive. Out of the 54 culture isolates,32 (59.26\%)were dermatophytes followed by yeasts 13 (24.07\%) and non dermatophytemoulds 9 (16.67\%). Among the dermatophytes,T.rubrum18 (33.33\%) was the most common followed by T.mentagrophyte $8(14.81 \%)$ and T.tonsurans 4 (7.41\%).Among the yeasts, C.albicans11 $(20.37 \%)$ was predominate followed by C.tropicalis 2 (3.7\%).Among the non dermatophytemoulds,Aspergillusflavus3 (5.55\%) was the commonest followed by Fusarium spp 2 (3.7\%).The finger nails were more commonly involved 66 (55\%) than toe nails $54(45 \%)$. Most of the patients were farmers 38 (31.67\%) followed by housewives 32(26.67\%).

CONCLUSION: Dermatophytes remain the predominant cause of onychomycosis, with T.rubrum as the most common aetiological agent, but less commonly yeasts like C.albicans and non dermatophytic moulds like Aspergillus spp can also cause nail infection and hence accurate diagnosis is needed, since the treatment is different for each group.

KEYWORDS: Onychomycosis, Dermatophytes, Trichophyton rubrum, Candida albicans

INTRODUCTION: Onychomycosis is common infection in adults and account for $20 \%$ of all nail diseases ${ }^{1}$.The factors that increase the prevalence of onychomycosis include increasing age, male sex, underlying conditions such as diabetes and immunodeficiency ${ }^{2}$.Although not life 
threatening, this may have significant clinical consequences such as secondary bacterial infections, chronicity, therapeutic difficulties and disfigurement in addition to acting as a reservoir of infection. ${ }^{3}$ The symptomatic disease can be a source of embarrassment and potential cause of morbidity ${ }^{4}$. Onychomycosis can be classified Into several clinical types: Distal and lateral subungual onychomycosis, proximal subungual onychomycosis, white superficial onychomycosis and Total dystrophic onychomycosis ${ }^{1}$. Most of the cases are due to dermatophytes, but yeasts and non dermatophytic fungi can also cause nail infection, particularly after trauma or diseases causing nail dystrophy. ${ }^{1}$ The clinical presentation may often be confused with other conditions like psoriasis, lichenplanus, onychodystrophy and nail trauma ${ }^{1}$, making laboratory diagnosis and confirmation necessary. Fungal cultures are essential for accurate identification of the causative organism. This is of paramount importance because the clinical outcome of antifungal agents varies as to whether the aetiological agent is a dermatophyte, yeast or a non-dermatophytic mould. ${ }^{5}$

The present study was undertaken to isolate and identify the aetiological agents of onychomycosis.

MATERIAL AND METHODS: The present study of onychomycosis was carried out in the department of Microbiology, S. S. Institute of medical sciences and research centre. A total of one hundred and twenty clinically diagnosed randomly selected cases of nail infection, of all age groups and of both sexes, attending Dermatology and Venereology outpatient department of S. S. hospital, Davangere, were studied. A detailed history of selected cases was taken in relation to name, age, sex, address, occupation and involvement of more than one site. Patients under antifungal treatment were excluded from the study group.

The affected area was cleansed with $70 \%$ ethyl alcohol and the nail specimen was collected by taking clippings of the infected part and scrapings beneath thenail $1{ }^{1,6}$. One portion of the specimen was subjected to $20 \%$ potassium hydroxide $(\mathrm{KOH})$ wet preparation for 1 to 2 hours at room temperature in a moist chamber ${ }^{7-9}$, and the remaining material was inoculated onto two sets of test tubes, one containing Sabouraud's dextrose agar with $0.05 \%$ chloramphenicol and the other containing Sabouraud's dextrose agar with $0.05 \%$ chloramphenicol and $0.5 \%$ cycloheximide and incubated at $28^{\circ} \mathrm{C}$ for up to 4 weeks. ${ }^{6,7,9}$ Cultures were read initially at 24 to48 hours for non dermatophytes and then on periodically for up to 4 weeks for dermatophytes. If no growth was found after 4 weeks, it was taken as negative for the growth of fungi and discarded. Repeat cultures were performed in cases where culture was negative for dermatophytes but positive for non dermatophytic moulds or yeasts to rule out the possibility of contamination.710,11 The criteria used to report non dermatophytic moulds or yeasts as pathogens was direct microscopy positive and isolation of same fungus in three consecutive samples at intervals of 7 days. ${ }^{11}$

Fungal isolate was identified based on colony morphology, reverse pigmentation, growth rate, microscopy (LPCB), slide culture and special tests like hair perforation test, urease test, germ tube test, sugar fermentation and assimilation tests $1,6,8,12$.

RESULTS: A total of 120 patients with clinical suspicion of onychomycosis were included in the study. Most of the patients were farmers 38 (31.67\%) followed by house wives $32(26.67 \%)$.

The finger nails were more frequently involved 66 (55\%) than toe nails 54 (45\%).

Out of 120 clinically suspected cases of onychomycosis, fungi were demonstrated in 66 (55\%) either by direct microscopy and / or culture. Forty Eight (40\%) were positive by both 
microscopy and culture. Twelve (10\%) were positive by microscopy and negative by culture. Six (5\%) were negative by microscopy but culture positive. Fifty four (45\%) were negative both by microscopy and culture.

Overall out of the 54 culture isolates, 32 (59.26\%) were infected with dermatophytes, followed by Candida species 13 (24.07\%) and non dermatophytemoulds $9(16.67 \%)$.

Various fungi isolated is shown in table 3.

DISCUSSION: In the present study,120 clinically suspected cases of onychomycosis attending Dermatology and Venereology out patient department of S. S. hospital, Davangere were studied.

In the present study, Males(60\%) were more commonly affected than females(40\%), which is comparable with the studies of Adhikari L et al ${ }^{14}$,NeupaneS et $\mathrm{al}^{2}$, where as Madhuri JT et $\mathrm{al}^{9}(51.96 \%)$ and Bokhari et $\mathrm{al}^{8}(72 \%)$ have reported higher prevalence in females. Male predominance may be due to increased outdoor physical activity and increased opportunity for exposure. Toe nail infection(52.78\%) was commoner in males, while finger nail infection(66.67\%) was common in females. This may be due to increased exposure to wet work in females, as most of them were house wives.

Onychomycosis was most commonly seen in farmers(31.67\%), followed by house wives $(26.67 \%)$, where as students were least affected $(9.17 \%)$, which is comparable with the study of Veer $\mathrm{P}$ et $\mathrm{al}^{10}$, where as NeupaneS et $\mathrm{al}^{2}$ reported that students were more commonly affected(31.3\%) followed by house wives(28.5\%).High prevalence in farmers and house wives may be due to increased outdoor physical activity and increased exposure to wet work respectively. It is less frequently seen in students due to rapid growth of nails which causes elimination of dermatophytes.

In the present study, out of 120 clinically suspected cases of onychomycosis, fungus was demonstrated in 66 (55\%) either by direct microscopy and/or culture. Direct microscopy was positive in 60 (55\%) and culture in 54 (45\%), which is comparable with the studies of Madhuri JT et $\mathrm{al}^{9}$ and Das NK et al ${ }^{12}$, where as Malik NA et al7has reported only $16 \%$ culture positivity. This variation in direct microscopy and culture may be due to non viability of fungal elements in some cases.

Out of the 54 fungal isolates, dermatophytes 32 (59.26\%) were the commonest, followed by yeasts $13(24.07 \%)$ and non dermatophytic moulds 9 (16.67\%).Among the dermatophytes, T.rubrum was the predominate isolate 18 (33.33\%) followed by T.mentagrophyte $8(14.81 \%)$,T.tonsurans $4(7.41 \%)$ and E.floccosum 2 (3.7\%).

Among the yeasts, C.albicans11 (20.37\%) was the most common followed by C.tropicalis $2(3.7 \%)$ and most of them were females due to immersion of hands frequently in water.

Among the non dermatophyticmouldsisolated,A.flavus3 (5.55\%) was the most common followed by Fusariumspp2 (3.7\%).A.niger and curvularia were isolated one each(1.85\%) and two isolates(3.7\%) could not be identified. This is comparable with the studies of Das NK et al'12, Veer $\mathrm{P}$ et $\mathrm{al}^{10}$ and Malik NA et $\mathrm{al}^{7}$, where as Vijaya D et al ${ }^{11}$ and Adhikari L et al ${ }^{14}$ reported yeasts and T.tonsurans as predominant fungi respectively in their study.

CONCLUSION: Onychomycosis is very common in our country where hot and humid climate in association with poor hygienic conditions play an important role in the growth of fungi and also majority of the people are agriculturists. It can no longer be considered as simple cosmetic problem, but can considerably impair patients quality of life. 
Dermatophytes remain the predominant cause of onychomycosis, with T.rubrum as the most common aetiological agent, but less commonly yeasts like C.albicans and non dermatophytic moulds like Aspergillus spp can also cause nail infection and hence accurate diagnosis is needed, since the treatment is different for each group.

\section{REFERENCES:}

1. Chander J(2009).Text book of Medical mycology.3rded.Newdelhi:Mehta publisher;pg:132-4, 56-63.

2. Neupane S, Pokhrel DB and PokhrelBM.onychomycosis:Aclinicoepidemiologicalstudy.Nepal Med Coll J 2009;11(2):92-95.

3. ErbaqciZ, TuncelA,Zer $Y$ et al. A prospective epidemiological survey on the prevalence of onychomycosis and dermatophytosis in male boarding school residents.Mycopathologica 2005;159:347-52.

4. GuptaAK,TabordaP,etal.Epidemiology and prevalence of onychomycosis in HIV positive individuals.Int J Dermatol 2000;39:746-53.

5. RamaniR,Srinivas CR, et al.Moulds in onychomycosis.Int J Dermatol 1993;32:877-8.

6. ColleeJG,Fraser AG, et al(1996).Mackie and McCartney practical medical microbiology.14thed.Edinburgh:Churchhill livingstone:pg:695-714.

7. MalikNA,Raza N, et al.Nondermatophytemoulds and yeasts as causative agents in onychomycosis.Journal of Pakistan Association of Dermatologists 2009;19:74-78.

8. Bokhari MA et al. onychomycosis in Lahore.PakistanInt J Dermatol 1999;38:5915.

9. MadhuriJT,Rama RGR, et al.Onychomycosis:A significant medical problem.Indian J DermatolVenereol Leprol2002;68(6):326-9.

10. Veer $P$ et al.A study of onychomycosis:Prevailing fungi and pattern of infection. Indian J Med Microbiol2007;25:53-6.

11. Vijaya D ,Anandkumar BH, et al. Study of onychomycosis. Indian J DermatolVenereolLeprol 2004;70(3):185-6.

12. DasNK,Ghosh P, et al.A study on the aetiological agent and clinico-mycological correlation of finger nail onychomycosis in eastern india. Indian J Dermatol 2008;53:75-9.

13. VelezA,Linares MJ et al.study of onychomycosis in Corboda,Spain:Prevailing fungi and pattern of infection.Mycopathologica 1997;137:1-8.

14. Adhikari L, Gupta AD et al.Clinico-aetiologic correlates of onychomycosis in Sikkim.Indian J PatholMicrobiol 2009;52:194-7.

Table 1 shows occupational status of the study group.

Journal of Evolution of Medical and Dental Sciences/Volume1/Issue5/November-2012Page-826 
ORIGINAL ARTICLE

\begin{tabular}{|c|c|c|}
\hline Occupation & Frequency & Percentage \\
\hline Farmers & 38 & $31.67 \%$ \\
\hline Housewives & 32 & $26.67 \%$ \\
\hline Office workers & 13 & $10.83 \%$ \\
\hline Students & 11 & $9.17 \%$ \\
\hline Miscellaneous & 26 & $21.67 \%$ \\
\hline
\end{tabular}

Table 2 shows nail involvement according to gender.

\begin{tabular}{|l|l|l|l|}
\hline \multirow{2}{*}{ Gender } & \multicolumn{2}{|c|}{ site } & \multirow{2}{*}{ Total } \\
\cline { 2 - 4 } & fingernail & Toe nail & \\
\hline Male & $34(47.22 \%)$ & $38(52.68 \%)$ & 72 \\
\hline Female & $32(66.67 \%)$ & $16(33.38 \%)$ & 48 \\
\hline Total & 66 & 54 & 120 \\
\hline
\end{tabular}

Various fungi isolated is shown in table 3.

\begin{tabular}{|l|c|c|c|c|}
\hline Causative agent & Finger nail & Toe nail & Total & percentage \\
\hline Dermatophytes & 10 & 8 & 18 & $33.33 \%$ \\
T. rubrum & 5 & 3 & 8 & $14.81 \%$ \\
T. mentagrophyte & 2 & 2 & 4 & $7.41 \%$ \\
T. tonsurans & 0 & 2 & 2 & $3.70 \%$ \\
E. floccosum & & & & \\
\hline Yeasts & 1 & 3 & 11 & $20.37 \%$ \\
C. albicans & 1 & 2 & $3.70 \%$ \\
C. tropicalis & 1 & 2 & 3 & $5.55 \%$ \\
\hline Non-dermatophytes & 1 & 1 & 2 & $3.70 \%$ \\
A. flavus & 1 & 0 & 1 & $1.85 \%$ \\
Fusariumspp & 1 & 0 & 1 & $1.85 \%$ \\
Curvulariaspp & 0 & 2 & 2 & $3.70 \%$ \\
A. niger & $\mathbf{3 0}$ & $\mathbf{2 4}$ & $\mathbf{5 4}$ & $\mathbf{1 0 0 \%}$ \\
unidentified & & & & \\
\hline \multicolumn{1}{|c|}{ Total } & & & 3 & \\
\hline
\end{tabular}



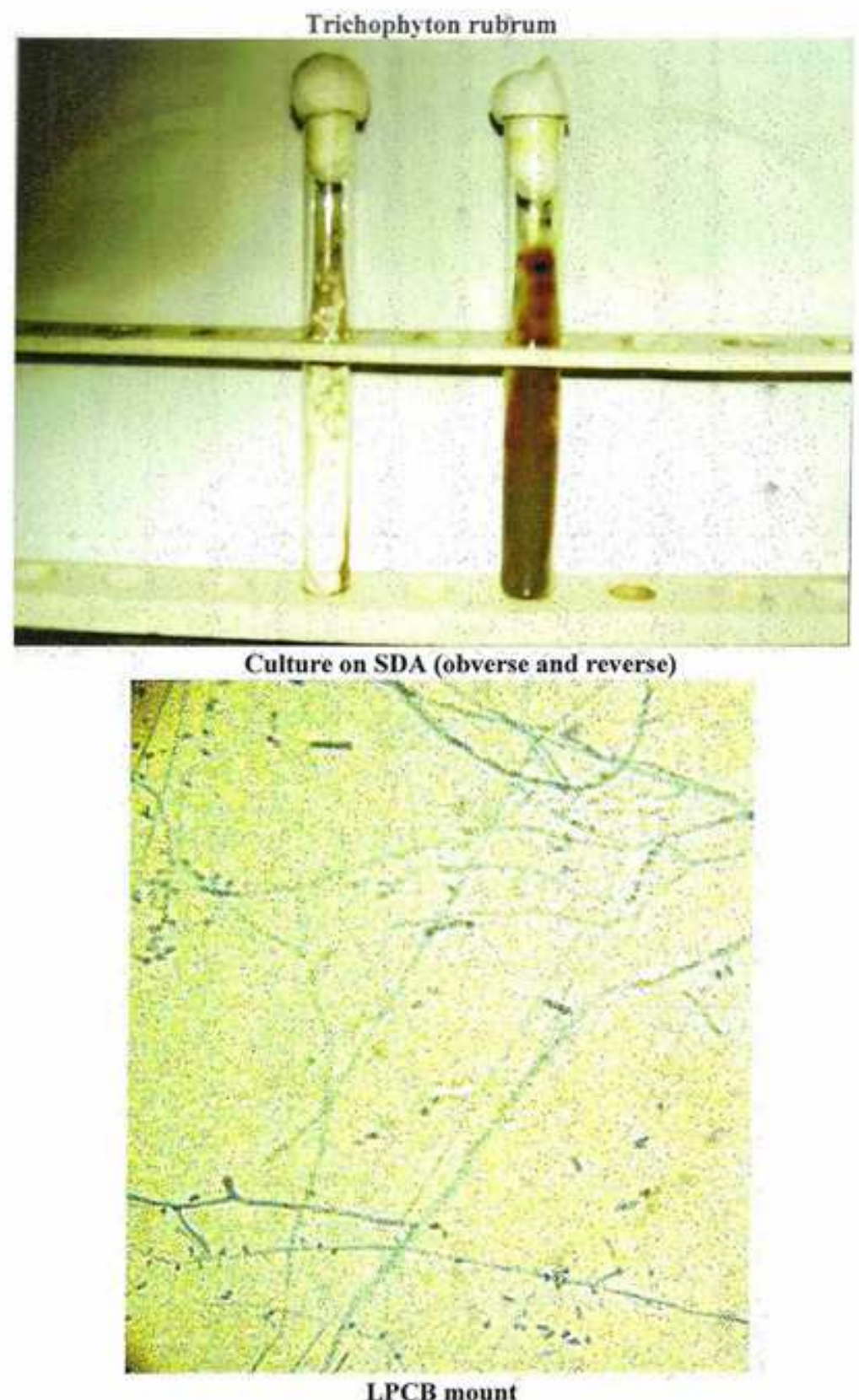

LPCB mount 


\section{ORIGINAL ARTICLE}

\section{Trichophyton mentagrophytes}

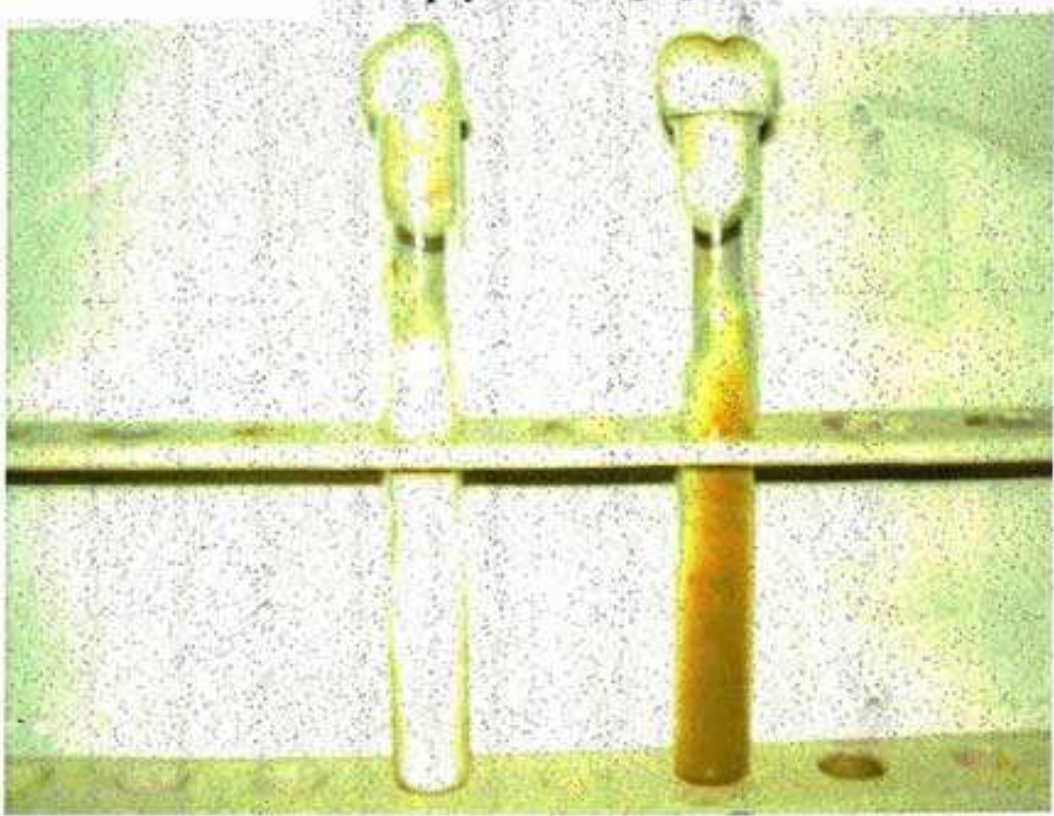

\section{Culture on SDA (obverse and reverse)}

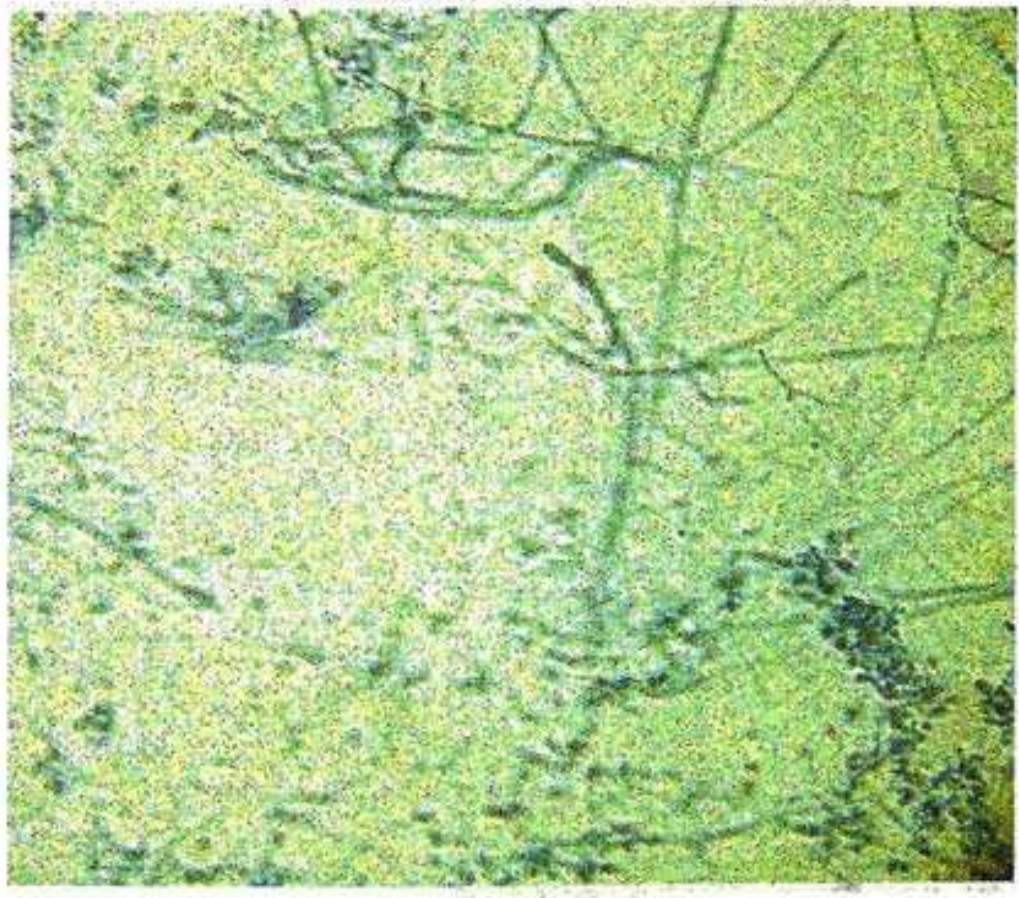

LPCB mount 